1. die direkte Anregung durch Stoß zwischen im elektrischen Feld beschleunigten "heißen“ Elektronen und $\mathrm{Mn}^{2+}$-Zentren,

2. die Übertragung der beim Übergang von Elektronen zwischen Störstellen und Bändern freiwerdenden Energie durch Resonanz an $\mathrm{Mn}^{2+}$. Zentren,

3. die Reabsorption der Emission von $\mathrm{Cu}$-Zentren in $\mathrm{Mn}^{2+}$-Zentren.

Im ersten Fall dürfte eine verzögerte Rekombination nicht beobachtet werden. Im zweiten Fall müßte sich die Mn-Emission einordnen lassen in das System anderer Übergangselemente, die Niveaus in der verbotenen Zone bilden. Im letzten Falle müßten die Spannungskurven der gelben bzw. grünen und blauen Emission streng parallel liegen.
Das Auftreten von zwei Steigungskonstanten $C$ oberhalb und unterhalb von $U_{0}$ in der Spannungscharakteristik der Mn-Emission zeigt, daß mindestens zwei verschiedene Mechanismen vorliegen, die allerdings noch nicht sicher identifiziert werden können.

In einer folgenden Arbeit soll das Problem des Energietransportes zwischen unterschiedlichen Rekombinationszentren in der Elektrolumineszenz reaktionskinetisch behandelt werden ${ }^{25}$.

Wir danken Herrn Prof. Dr. I. Broser für zahlreiche wertvolle Diskussionen und der Deutschen Forschungsgemeinschaft sowie der Verwaltung des ERP-Sondervermögens für die materielle Unterstützung dieser Arbeit.

25 I. Broser, H.-E. Gumlich u. R. Moser, Z. Naturforschg. 20 a [1965], im Druck.

\title{
Zur Theorie nichtlinearer Spinorfelder
}

\section{H. Mitrer}

\author{
Max-Planck-Institut für Physik und Astrophysik, München
}

(Z. Naturforschg. 20 a, 1505-1518 [1965] ; eingegangen am 30. Juli 1965)

\begin{abstract}
The formal structure of a relativistic field theory is examined using functional techniques for GrEen's functions. The consequences of a locally conserved current constructed from a nonlocal bilinear covariant are studied. They result in a modified from of the Takahashi identities. Some problems are briefly discussed, which arise, if one tries to match conventional techniques as e.g. the Bethe-Salpeter-method or the Schwinger-Fradkin formal solution with noncanonical quantisation. The consistency of some approximation methods in relation to the afore mentioned problems and to the existence of local currents is investigated. An expansion of the mass operator in powers of the interaction, using exact propagators, turns out to be consistent only with canonical quantisation. For $\mathrm{T}_{\mathrm{AMM}}-\mathrm{D}_{\mathrm{ANCOF}}-\mathrm{like}$ approximations the problem is more intricate.
\end{abstract}

\section{Einleitung und Problemstellung}

Die relativistische Quantentheorie wechselwirkender Felder weist eine sehr komplizierte Struktur auf, so $\mathrm{da} ß$ es bisher nicht gelungen ist, exakte Lösungen in nichttrivialen Fällen anzugeben. Für die Diskussion von Näherungsverfahren ist es aber von Wichtigkeit, die formale Struktur einer Theorie so klar wie möglich vor Augen zu haben. Die folgende Arbeit verfolgt in erster Linie den Zweck, diese Struktur für eine Spinortheorie mit Selbstwechselwirkung herauszuarbeiten. Dadurch wird es möglich sein, eine übersichtliche Diskussion bereits verwendeter

1 H. P. DürR, W. Heisenberg, H. Mitter, S. Schlieder u. K. YAMAZAKI, Z. Naturforschg. 14 a, 441 [1959]. - H. P. DürR, ibid. 16 a, 327 [1961]. - Für eine zusammenfas-
Näherungsverfahren vorzunehmen und mögliche Verbesserungen anzugeben.

Wir betrachten die Feldgleichung

$$
D \psi+l^{2} Q^{v} \psi\left(\bar{\psi} Q^{v} \psi\right)=0 .
$$

$\psi$ sei dabei ein quantisiertes Spinorfeld, das der Vertauschungsrelation

$$
\{\psi(x), \bar{\psi}(y)\}=0 \quad \text { für }(x-y)^{2} \text { raumartig }
$$

genügt. $D$ sei ein invarianter Differentialoperator erster Ordnung, $Q$ seien geeignete Matrizen. Für die einfachste Form der von HeIsenberg und Mitarbeitern studierten Theorie ist z. B. ${ }^{1}$

sende Darstellung siehe W. Heisenberg, Introduction to the unified field theory of elementary particles, J. Wiley, New York, im Erscheinen. 


$$
\begin{gathered}
D=\frac{1}{i}\left(\sigma^{v} \frac{\partial}{\partial x^{\nu}}\right), \\
\underset{\substack{\alpha \beta^{\prime} \beta^{\prime} \\
Q_{\gamma}^{v}}}{Q_{\gamma^{\prime} \delta^{\prime}}^{v}=\frac{1}{4}}\left(3 \delta_{\alpha^{\prime} \beta^{\prime}} \delta_{\gamma^{\prime} \delta^{\prime}}+\boldsymbol{\tau}_{\alpha^{\prime} \beta^{\prime}} \boldsymbol{\tau}_{\gamma^{\prime} \delta^{\prime}}\right) \sigma_{\alpha \beta}^{u} \sigma_{\mu \gamma \delta} .
\end{gathered}
$$

In dieser Form hat der Operator die Eigenschaft (FIERZ-Symmetrie)

$$
Q_{\alpha \beta}^{v} Q_{\alpha^{\prime} \beta^{\prime}}^{v} Q_{\gamma^{\prime} \delta^{\prime}}^{v}=-Q_{\alpha^{\prime} \delta^{\prime}}^{v} Q_{\gamma^{\prime} \beta^{\prime}}^{v} .
$$

Unsere Überlegungen haben für andere Kopplungen ebenfalls Gültigkeit, sofern sie $(3 \mathrm{~b})$ erfüllen. Wir benützen für die Multiplikation im Spin- und Isospinraum und die Faltung im Koordinatenraum den Matrizenkalkül mit Summationsübereinkommen für doppelt vorkommende Indizes und schreiben die Differentialgleichung durch Einführung von

$$
D_{i k}=\delta\left(x^{(i)}-x^{(k)}\right) D
$$

und

$$
\begin{gathered}
V_{i k, l m}=l^{2} \delta\left(x^{(i)}-x^{(k)}\right) \delta\left(x^{(i)}-x^{(l)}\right) \\
\cdot \delta\left(x^{(i)}-x^{(m)}\right) \underset{v}{Q_{i}^{i l} l^{\prime}} Q_{k^{\prime} m^{r}}^{v}
\end{gathered}
$$

formal als nichtlokale Gleichung

$$
D_{i k} \psi_{k}+V_{i m, l n} \bar{\psi}_{m} \psi_{n} \psi_{l}=0 .
$$

Für andere Kopplungen als die durch (3) gegebene ist lediglich $(4 \mathrm{~b})$ entsprechend abzuändern.

Gl. (1) bzw. (5) sind als rein formale Beziehungen aufzufassen, solange über die Produktbildung von Feldoperatoren am selben Ort keine Aussage gemacht wird. Wir fassen dieses Problem als Konsistenzproblem auf: Wir betrachten das durch (5) induzierte System von Gleichungen für Vakuumerwartungswerte von zeitlich geordneten Produkten von Feldoperatoren (Greensche Funktionen), das wir (im allgemeinen näherungsweise) zu lösen versuchen. Aus der Struktur der Lösungen bei kleinen Abständen versuchen wir dann sinnvolle Modifikationen von $V_{i k, l m}$ zu finden, die die Form der Lösungen möglichst wenig ändern, so daß bei Fortsetzung dieser Prozedur schließlich eine konsistente Theorie entsteht.

\section{Erzeugendes Funktional, Green'sche Funktionen}

Die Gleichungen für die feldtheoretischen Greenschen Funktionen sind am besten mit Hilfe des Funktionalkalküls abzuleiten. Die Greenschen Funktionen werden dabei als Antwortfunktionen auf eine kleine äußere Störung aufgefaßt: sie sind dann im wesentlichen die Entwicklungskoeffizienten des Funk- tionals, das die Änderung des Grundzustandes unter dem Einfluß einer äußeren Störquelle beschreibt, nach Potenzen dieser Quelle. Ein für unsere Zwecke besonders geeigneter Formalismus wurde von BRENIG und WAGNER ${ }^{2}$ angegeben und in der nichtrelativistischen Theorie des Mehrteilchenproblems mit Erfolg angewendet, bei dem die Wechselwirkung ebenfalls durch eine Kopplung von vier Fermionfeldoperatoren beschrieben wird. Der Formalismus ist sofort auf den relativistischen Fall zu übertragen. Wir führen eine nicht lokale, an $\psi_{i} \bar{\psi}_{k}$ gekoppelte äußere Quelle ein und transformieren in die Wechselwirkungsdarstellung bezüglich der Kopplung an das äußere Störfeld, so daß die transformierten Feldoperatoren wieder die quellenfreie Gleichung (5) erfüllen. Als Greensche Funktionen erklären wir dann mit

$$
U=\exp \left\{i q_{l k} \psi_{k} \bar{\psi}_{l}\right\}, \quad V=\langle 0|T U| 0\rangle
$$

die Größen

$$
\begin{gathered}
G_{k l}=\frac{i}{V}\left\langle 0\left|T \psi_{k} \bar{\psi}_{l} \mathrm{U}\right| 0\right\rangle=\frac{\delta}{\delta q_{l k}} \ln V, \\
G_{k m, l n}=\frac{1}{V}\left\langle 0\left|T \psi_{k} \psi_{m} \bar{\psi}_{l} \bar{\psi}_{n} U\right| 0\right\rangle .
\end{gathered}
$$

Die physikalisch interessanten Größen sind dann die Grenzwerte der Greesschen Funktionen für verschwindende äußere Quellen

$$
\begin{aligned}
S_{k l} & =\lim _{q \rightarrow 0} G_{k l}, \\
S_{k l, m n} & =\lim _{q \rightarrow 0} G_{k l, m n} \quad \text { etc. }
\end{aligned}
$$

Sie werden, wie aus (7) zu sehen ist, durch das Funktional $V$ erzeugt, d. h. sie entstehen aus ihm durch ein- oder mehrmalige Differentiation nach der Quelle und Nullsetzen derselben. So erhält man z. B. aus der Definition (7) eine Darstellung für eine Korrelationsfunktion als Funktionalableitung

$$
\begin{aligned}
F_{k m, l n} \equiv G_{k m, l n}- & G_{k l} G_{m n} \\
& =\frac{\delta G_{k l}}{\delta q_{n m}}=\frac{\delta}{\delta q_{n m}} \frac{\delta}{\delta q_{l k}} \ln V .
\end{aligned}
$$

An Stelle dieser Korrelationsfunktion ist für manche Zwecke die Verwendung „amputierter" Größen von Vorteil. Durch Abspalten von zwei Faktoren $G$ (Amputation von zwei Beinen) erhält man eine oft verwendete Größe, die Vertexfunktion

$$
\begin{aligned}
& F_{i p, j q}=-G_{i k} \Gamma_{k p, l q} G_{l j}, \quad \mathcal{E}=\oint^{\delta} \\
& 2 \text { W. BRenig u. H. WaGNER, Z. Phys. 173, } 484 \text { [1963]. }
\end{aligned}
$$


die, wie man sich leicht überzeugt, die Funktionalableitung der reziproken Ausbreitungsfunktion ist

$$
\Gamma_{i p, j q}=\delta G_{i j}^{-1} / \delta q_{q p}
$$

Für gewisse Probleme ist es günstiger, von dem korrelierten Anteil von $F$

$$
\eta_{i k, l m}=G_{i k, l m}-G_{i l} G_{k m}+G_{i m} G_{k l}=F_{i k, l m}+G_{i m} G_{k l}
$$

auszugehen. Wir definieren zwei durch Amputation entstehende Größen durch

$\eta_{i s, k r}=-L_{i s, j r} G_{j k}=i G_{i j} G_{s m} T_{j m, l n} G_{l k} G_{n r}$.

Die Zweckmäßigkeit dieser Definitionen wird später einsichtig werden.

Die Massen von Teilchen-Antiteilchen-Bindungszuständen und die Ankopplung derselben können aus der Struktur der Vierpunktfunktionen berechnet werden. Sei die Fourier-Transformation von $F$

$$
F_{i k, j l}=\frac{1}{(2 \pi)^{12}} \mathrm{~d}^{4} p \mathrm{~d}^{4} q \mathrm{~d}^{4} k \exp \left\{i\left(k / 2, x^{i}+x^{j}-x^{k}-x^{l}\right)\right\} \exp \left\{i\left(p, x^{i}-x^{j}\right)\right\} \exp \left\{i\left(q, x^{k}-x^{l}\right)\right\} F_{i k, j l}(p, q \mid k)
$$

(analog für $\Gamma, \eta$ oder $L$ ), so kann gezeigt werden ${ }^{3}$, daß $F$ bei Vorhandensein von Bindungszuständen Pole in der Variablen $k^{2}$ hat, in deren Umgebung die Bilinearentwicklung

$$
\begin{gathered}
F_{i k, j l}(p, q \mid k)=\sum_{n} \frac{\varphi_{i j}(p, k) \varphi_{k l}(q, k)}{k^{2}+M_{n}{ }^{2}}+\ldots \\
\qquad=\Sigma{ }^{+\cdots}
\end{gathered}
$$

gilt. Da der unkorrelierte Anteil an dieser Stelle regulär ist, kann er vernachlässigt werden, und alle anderen Vierpunktfunktionen $(T, \eta, L, T)$ haben ein analoges Verhalten. Die Kopplungskonstanten für die gebundenen Teilchen können aus den Residuen von $\Gamma$ an den entsprechenden Polen berechnet werden. Man hat dabei aber zu beachten, daß nicht alle Pole als Teilchen (echte Bindungszustände) interpretierbar sind. Infolge der singulären Struktur der Integralgleichungen, denen die Vierpunktfunktionen in relativistischen Theorien genügen, können, vor allem bei der Ruhemasse Null, "anomale" Pole auftreten, die pathologische Eigenschaften haben (z. B. Kopplungskonstante Null, unendliche oder negative Norm) und daher keine physikalische Bedeutung haben ${ }^{4}$. Ihre Interpretation als Teilchen würde zu Schwierigkeiten (nichtunitäre $S$ Matrix) führen.

3 S. Mandelstam, Proc. Roy. Soc. London A 233, 248 [1955].

4 Siehe J. Goldstern, Phys. Rev. 91, 1516 [1953], ferner S. Mandelstam, 1. c. ${ }^{3}$ und Proc. Roy. Soc. London A 237, 496 [1956]. Ähnliche Verhältnisse liegen für die Drrac-Gleichung vor: K. M. CASE, Phys. Rev. 80, 797 [1950]. Manchmal haben Pole, die von verletzten Symmetrien herrühren (sog. „Goldstone-Teilchen"), diese Eigenschaft, s. M. BAker, K. Johnson u. B. W. Lee, Phys. Rev. 133, B 209 [1964] und K. Johnson, nicht veröffentlicht.

\section{Gleichungen für die Green'schen Funktionen}

Als Folge von (1) bzw. (5) erfüllen die GreenFunktionen ein gekoppeltes, unendliches System von Differentialgleichungen, das durch die Funktionaldifferentialgleichung

$$
\left(D_{k l}+\varrho_{0} q_{k l}+i V_{k r, l s} \frac{\delta}{\delta q_{s r}}\right) \frac{\delta}{\delta q_{m l}} V=\varrho_{0} \delta_{k m} V
$$

erzeugt wird. Dabei ist $\varrho_{0}$ das niedrigste Moment der Lehmansschen Spektralfunktion von $G$ :

$$
\varrho_{0}=\int_{0}^{\infty} \varrho\left(\varkappa^{2}\right) \mathrm{d} \varkappa^{2} .
$$

Ihre Kenntnis impliziert eine Aussage über das Verhalten der Theorie bei kleinen Abständen, die an dieser Stelle noch nicht gemacht werden soll. Bei kanonischer Quantisierung wird $\varrho_{0}=1$ gesetzt. Es kann sich herausstellen, daß dies nicht widerspruchsfrei möglich ist und nur $\varrho_{0}=0$ zu einer divergenzfreien Theorie führt (nichtkanonische Quantisierung). Bei anwesendem Feld wird $\varrho_{0}$ selbst im letzteren Fall nicht notwendig Null sein. Damit die hier gegebene Herleitung gilt, muß dann jedoch

$$
\lim _{q \rightarrow 0} \varrho_{0}=\lim _{q \rightarrow 0} \frac{\delta \varrho_{0}}{\delta q}=0
$$

angenommen werden, da Ableitungen von $\varrho_{0}$ vernachlässigt $\operatorname{sind}^{5}$.

\footnotetext{
5 Wenn der kanonische Vertauschungsausdruck für $\psi$ keine $c$-Zahl ist, muß in allen Formeln $\varrho_{0} \delta_{k m}$ durch $\gamma_{0} \delta\left(t_{k}-t_{m}\right)\left\langle 0\left|T\left\{\psi_{k}, \bar{\psi}_{m}\right\} U\right| 0\right\rangle \cdot V^{-1}$

ersetzt werden.
} 
Zur Illustration schreiben wir die untersten Gleichungen des Systems an. Für die Zweipunktfunktion erhält man

$$
\left(D_{k l}+\varrho_{0} q_{k l}+i V_{k r, l s}\left(G_{s r}+\frac{\delta}{\delta q_{r s}}\right)\right) G_{l m}=\varrho_{0} \delta_{k m} .
$$

Durch Einsetzen von (10) kann die Gleichung in eine Beziehung zwischen $G$ und $F$ (oder $T$ etc.) umgeschrieben werden. Für diese Größen kann man dann durch Differentiation von (19) weitere Gleichungen bekommen. Wir schreiben das System für $G$ und $L$ aus (14) an:

$$
\begin{gathered}
\left(D_{k l}+\varrho_{0} q_{k l}+i V_{k r, j s}\left(2 \delta_{j l} G_{s r}-L_{j s, l r}\right)\right) G_{l m}=\varrho_{0} \delta_{k m}, \\
\left(D_{k l}+\varrho_{0} q_{k l}\right) L_{l b, n a}+2 i V_{k r, l s}\left(G_{s r} L_{l b, n a}+\delta_{l n} G_{i r} L_{s b, i a}\right) \\
+i V_{k r, l s}\left(\frac{\delta L_{l s, n r}}{\delta q_{a b}}-L_{l s, i r} L_{i b, n a}\right)
\end{gathered}
$$

Der Faktor 2, der in (20) im Vergleich zu (19) auftritt, hängt mit der Tatsache zusammen, daß $L$ aus der Korrelationsfunktion $\eta$ gewonnen wurde. Daher tritt im Wechselwirkungsterm nicht nur der Hartree-Term [wie z. B. in (19)], sondern auch der Fоск-Term auf, der hier wegen der Symmetrie. eigenschaft $(3 \mathrm{~b})$ mit dem HartreE-Term identisch ist.

\section{IV. Äquivalenz von Theorien mit Fermi- und Yukawa-Kopplung}

Wie von mehreren Autoren bemerkt wurde ${ }^{6}$, sind Theorien wie die hier betrachtete unter gewissen Umständen weitgehend äquivalent zu solchen wechselwirkender Bose- und Spinorfelder mit YukawaKopplung der Bose-Felder an die Fermionenströme. Da dieser Zusammenhang wichtig erscheint, soll er hier aufgezeigt werden.

Wir definieren eine Größe $a_{k l}$ durch

$$
a_{k l}=\varrho_{0} q_{k l}+i V_{k r, l s} G_{s r} .
$$

Dann ist wegen (10)

$$
\frac{\delta a_{k l}}{\delta q_{p q}}=\varrho_{0} \delta_{k p} \delta_{l q}+i V_{k r, l s} F_{s q, r p} \equiv D_{k q, l p} .
$$

Nun eliminieren wir die Ableitung nach $q$, indem wir alle Größen als Funktionale von $a$ auffassen:

$$
\frac{\delta}{\delta q_{r s}}=\frac{\delta a_{p q}}{\delta q_{r s}} \frac{\delta}{\delta a_{p q}}=D_{p s, q r} \frac{\delta}{\delta a_{p q}} .
$$

Wir gehen von (19) aus und schreiben für

$\frac{\delta G_{l m}}{\delta q_{r s}}=-G_{l l^{\prime}} \frac{\delta G_{l \prime m}^{-1}}{\delta q_{r s}} G_{m^{\prime} m}=-G_{l l^{\prime}} D_{p s, q r} \frac{\delta G_{l l^{\prime}}^{-1}}{\delta a_{p q}} G_{m^{\prime} m}$.

${ }^{6}$ I. Bialynicki-Birula, Phys. Rev. 130, 465 [1963] u. Rochester Report URPA-11 [1963]. - J. D. BJoRken, Ann. Phys. N. Y. 24, 174 [1963]. - G. S. Guralnik, Phys. Rev. 136, B 1404 u. B 1417 [1964]. - Die letzteren bei-
Definieren wir nun einen neuen Vertex durch

$$
\frac{\delta G_{l m}^{-1}}{\delta a_{a b}}=\gamma_{l b, m a},
$$

so wird (19)

$$
\left(D_{k l}+a_{k l}-i V_{k r, i s} G_{i j} D_{p s, q r} \gamma_{j q, l p}\right) G_{l m}=\varrho_{0} \delta_{k m} .
$$

Diese Gleichung ist vollkommen analog zu der entsprechenden Gleichung einer Boson-Fermion-Theorie mit Yukawa-Kopplung (in einem äußeren BoseFeld), wobei in letzterer $a_{k l}$ der Vakuumerwartungswert des Bose-Feldoperators (der bei Anwesenheit eines äußeren Feldes nicht verschwindet), $D$ der Bosonpropagator und $\gamma$ der Fermion-Boson-Vertex ist. Für die Elektrodynamik lautet die entsprechende Gleichung z. B.

$$
\left[i \gamma^{\mu} \partial_{\mu}-m+e_{0} \gamma^{\mu}\left\langle A_{\mu}\right\rangle-i e_{0} \gamma_{\mu} D^{\mu \nu} G \Gamma_{\nu}\right] G=\delta .
$$

Die Identifizierung von $D$ als Propagator ist auch in unserem Fall gerechtfertigt, wenn $F$ im Impulsraum Pole in $k^{2}$ hat, denn diese Variable entspricht, wie man sich durch Fourier-Transformation von (23) überzeugt, gerade dem Impuls des transportierten „Bosons“. Die Diskussion der Äquivalenz läuft also auf den Nachweis der entsprechenden Pole hinaus. Die Kopplungskonstanten sind im Prinzip aus (26) zu berechnen. Wenn $F$ bekannt ist, kennt man auch $\gamma$, denn diese Größe kann (wenigstens im Prinzip) durch Vergleich von (12) mit (25) unter Verwendung von (24), (23) und (11) berechnet werden.

den Autoren betrachten Theorien mit entartetem Vakuum. Siehe auch ältere Versuche von B. Jouver, Nuovo Cim. 5, I [1957]. - P. G. O. Freund, Acta Phys. Austr. 14, 445 [1961]. 


\section{Eichinvarianz und lokale Stromerhaltung}

In der zur Feldgleichung (5) gehörigen klassischen Theorie würde man in üblicher Weise auf die Existenz von lokalen Strömen schließen können, die aus Feldoperatoren am selben Ort bilinear gebildet sind:

$$
j^{\mu}(x)=\bar{\psi}(x) C^{\mu} B \psi(x)
$$

$\left(C^{\mu} B\right.$ ist dabei ein aus den Matrizen der Theorie gebildeter Ausdruck ${ }^{7}$ ) und als Konsequenz der Feldgleichung einer Kontinuitätsgleichung genügen:

$$
\partial{ }^{\mu} j_{\mu}=0 \text {. }
$$

In der Quantentheorie ist diese Schlußweise wegen der Singularität des Produktes von Feldoperatoren am selben Ort nicht zulässig. Man sieht jedoch, daß die Frage der Existenz lokaler Ströme mit der genauen Form des in (5) auftretenden Produktes zu tun hat. Von der Physik her gesehen ist nur die Existenz eines einzigen lokalen Stromes (des elektro- magnetischen) gesichert. Ob der zweiten absolut erhaltenen Ladung (der Baryonenzahl) in derselben Weise wie der elektromagnetischen ein lokaler Strom zugeordnet werden kann, ist zweifelhaft. Für die nur näherungsweise erhaltenen Größen (wie z. B. den Isospin) erscheint eine solche Annahme nicht sinnvoll. Unabhängig von dieser Problematik ist aber die Frage interessant, wann eine durch eine Vorschrift für das Produkt am selben Ort gegebene Theorie des hier betrachteten Typus einen lokalen Strom enthält. In diesem Abschnitt soll daher angenommen werden, daß dies der Fall ist, und es sollen die Konsequenzen der Annahme für die Greenschen Funktionen untersucht werden. Diese Konsequenzen sind für eine - exakt oder näherungsweise - vorgegebene Theorie einfacher zu überprüfen als die Operatorbeziehung (28). Besteht man im Rahmen einer Näherung darauf, sie Schritt für Schritt zu erfüllen, so kann man auch über mögliche Produktbildungen Aussagen machen, da dann jedenfalls das in (28) auftretende Produkt erklärt ist.

Wir betrachten die spezielle Quelle

$$
q_{k l}=C^{\mu} B A_{\mu}^{\mathrm{e}}\left(\frac{x^{(k)}+x^{(l)}}{2}\right) f\left(x^{(k)}-x^{(l)}\right) \exp \left\{i \int_{x_{i}}^{x_{k}} \mathrm{~d} \xi o A_{\varrho}^{\mathrm{e}}(\xi) B\right\}
$$

und die Eichtransformation

$$
\psi(x) \rightarrow e^{i \lambda(x) B} \psi(x), \quad A_{\mu}^{\mathrm{e}}(x) \rightarrow A_{\mu}^{\mathrm{e}}(x)+\partial_{\mu} \lambda(x) .
$$

Der Quellterm $q_{k l} \psi_{l} \bar{\psi}_{k}$ ändert sich bei (30) nur um die Divergenz des Stromes

$$
j^{\mu}(x)=\int \bar{\psi}(x+z / 2) C^{\mu} B \exp \left\{i \int_{x-z / 2}^{x+z / 2} \mathrm{~d} \xi \varrho A_{\varrho}^{\mathrm{e}}(\xi) B\right\} \psi(x-z / 2) f(z) \mathrm{d}^{4} z .
$$

Setzt man daher die Änderung von $V$ bei (30) Null, so erhält man eine Beziehung, die dem Erhaltungssatz (28) für diesen Strom äquivalent ist. Bevor wir diese Variation berechnen, sollen noch einige Bemerkungen zu den in (29) auftretenden Größen gemacht werden.

Die invariante Funktion $f$ ist als Ausschmierungsfunktion zu verstehen, die wegen der singulären Natur des Produktes der Feldoperatoren eingeführt wurde. Formal folgt (28) aus der zu (5) gehörigen LagrangeFunktion nur für streng lokales $f$. Über die genaue Form von $f$ Angaben a priori zu machen, erscheint riskant: eine $\delta$-Funktion, die zu (27) zurückführt, wäre sicherlich zu naiv. Es können kompliziertere Limesbildungen nötig sein. Eine schon vor längerer Zeit untersuchte und neuerdings wieder diskutierte Möglichkeit $^{8}$ wäre z. B.

$$
f(z)=\lim _{\varepsilon \rightarrow 0} \delta(z-\varepsilon),
$$

wobei der Limes möglichst spät (z. B. nach Ableitung der Gleichungen für die Greenschen Funktionen) zu bilden ist. Eine wirklich saubere Bestimmung von $f$ ist nur zusammen mit der am Schluß von Abschn. I formulierten Konsistenzbetrachtung möglich.

\footnotetext{
${ }^{7}$ Für die Kopplung (3) hätte man zwei Ströme $C^{\mu} B=\sigma^{\mu} \cdot 1$ bzw. $C^{\mu} B=\sigma^{\mu} \cdot \tau$ die als Baryonen- bzw. Isospinstrom zu interpretieren wären.
}

8 P. A. Dirac, Proc. Cambridge Phil. Soc. 30, 150 [1934]. W. Heisenberg, Z. Phys. 90, 209 und 92, 692 [1934]. J. G. Valatin, Proc. Roy. Soc. London A 222, 93 und 228 [1954]. - J. Schwinger, Phys. Rev. Letters 3, 296 [1959]. 
Die Größe $A_{\mu}^{\mathrm{e}}$ ist als äußeres („klassisches“) Feld zu interpretieren. Die Annahme, daß es so ein Feld gibt, ist (sofern es einen erhaltenen Strom gibt) wohl unproblematisch, da es nur als formales Hilfsmittel dient. Weniger klar ist schon, ob im Exponenten von (29) wirklich $A_{\mu}^{\mathrm{e}}$ stehen muß oder ob man in (31) ein aus Feldoperatoren $\psi$ geeignet aufgebautes „inneres“ Feld $A_{\mu}$ einzusetzen hat [das natürlich die richtige Eicheigenschaft (30) haben muß]. Im letzteren Fall wäre der Strom kein bilinearer Ausdruck mehr, und es wären auch noch an anderen Stellen Modifikationen nötig. Der Einfachheit halber soll hier angenommen werden, daß man mit $A_{\mu}^{\mathrm{e}}$ auskommt [oder, was wohl gleichwertig ist, daß $A_{\mu}$ im Exponenten von (31) durch seinen Vakuumerwartungswert ersetzt werden darf].

Wir berechnen nun die durch eine infinitesimale Transformation (30) bewirkte Änderung von $V$. Sie setzt sich zusammen aus der durch die Transformation der $\psi$ verursachten Änderung

$$
\begin{gathered}
\delta_{\psi} V=\left\langle 0\left|T U \exp i\left[q_{k l}\left(i \lambda_{l} B \quad \psi_{l} \bar{\psi}_{k}-i \lambda_{k} \psi_{l} \bar{\psi}_{k} B\right)+\ldots\right]\right| 0\right\rangle-\langle 0|T U| 0\rangle \\
=-\left\langle 0\left|T U q_{k l}\left(\lambda_{l} B \psi_{l} \bar{\psi}_{k}-\lambda_{k} \psi_{l} \bar{\psi}_{k} \tilde{B}\right)\right|\right\rangle=-\frac{1}{i} q_{k l}\left(\lambda_{l}\left(B \frac{\delta}{\delta q}\right)_{k l}-\lambda_{k}\left(\frac{\delta}{\delta q} \tilde{B}\right)_{k l}\right) V, \\
\tilde{B}=C_{0} B^{+} C_{0}, \quad C_{\mu} B=\tilde{B} C_{\mu},
\end{gathered}
$$

und der durch die Änderung der Quelle bedingten V ariation

$$
\delta_{q} V=\frac{\delta V}{\delta q_{k l}} \delta q_{k l}=\int \mathrm{d}^{4} x_{(k)} \mathrm{d}^{4} x_{(l)} \frac{\delta V}{\delta q_{k l}} C^{\mu} B f\left(x_{(k)}-x_{(l)}\right) \partial_{\mu}\left(\frac{x_{(k)}+x_{(l)}}{2}\right) \lambda\left(\frac{x_{(k)}+x_{(l)}}{2}\right) .
$$

Setzt man die gesamte Änderung von $V$ Null $\quad \delta V=\delta_{\psi} V+\delta_{q} V=0$,

so erhält man nach einmaliger partieller Integration und Einführung der Schwerpunkts- und Relativkoordinaten

$$
x_{m}=\frac{1}{2}\left(x_{(k)}+x_{(l)}\right), z=x_{(k)}-x_{(l)}
$$

durch Koeffizientenvergleich bezüglich $\lambda$ die Beziehung

$$
\left(C^{\mu} B\right)_{k l} \partial_{\mu}\left(x_{m}\right) \int \mathrm{d}^{4} z \frac{\delta V}{\delta q_{k l}} f(z)=i q_{k l}\left\lceil\delta_{m l}\left(B \frac{\delta}{\delta q}\right)_{k l}-\delta_{m k}\left(\frac{\delta}{\delta q} \tilde{B}\right)_{k l}\right] V .
$$

Division durch $V$ zeigt, daß dieselbe Relation auch für $\ln V$ gilt.

Diese Beziehung erzeugt ein unendliches System von Beziehungen zwischen den Greenschen Funktionen, die sogenannten WARD-TAKAHASHI-Identitäten $^{9}$, die dem Erhaltungssatz (28) äquivalent sind. Zur Illustration geben wir die untersten beiden Relationen an. In nullter Ordnung bezüglich $q$ erhält man die Kontinuitätsgleichung für den Vakuumstrom

$$
\partial_{\mu}\left(x_{m}\right) \operatorname{Sp} C^{\mu} B \int S\left(x_{(k)}, x_{(l)}\right) f(z) \mathrm{d}^{4} z=0 .
$$

In erster Ordnung erhält man

$$
i\left(\delta_{m r}(B S)_{r s}-\delta_{m s}(S \tilde{B})_{r s}\right)=\left.\left(C^{\mu} B\right)_{k l} \partial_{\mu}(m) \int f(z) F_{r l, s k} \mathrm{~d}^{4} z\right|_{q=0} .
$$

Diese als verallgemeinerte $\mathrm{W}_{\mathrm{ARD}}$-Identität bekannte Beziehung kann auch als Relation zwischen $S^{-1}$ und $\Gamma$ geschrieben werden:

$$
\left(\delta_{m r}\left(\tilde{B} S^{-1}\right)_{r s}-\delta_{m s}\left(S^{-1} B\right)_{r s}\right)=\left.\left(C^{\mu} B\right)_{k l} \frac{1}{i} \partial_{\mu}(m) \int \mathrm{d}^{4} z f(z) \Gamma_{r l, s k}\right|_{q=0} .
$$

Für eine vorliegende Näherungslösung können die WARD-TAKAHASHI-Identitäten, z. B. (36), stets nachgeprüft werden. Die Näherung wird nur dann mit der Existenz eines lokalen Stromes und mit der

9 Diese Relationen wurden in der Feldtheorie bisher nur für lokal aufgebaute Stromausdrücke diskutiert, vgl. Y. TAKAhashi, Nuovo Cim. 6, 370 [1957]. Ein Spezialfall von (28) wurde für die Elektrodynamik schon vorher störungstheo-
Ausgangsgleichung (5) verträglich sein, wenn die Identitäten mit nicht zu stark nichtlokaler Ausschmierung erfüllt sind, so daß die verbleibende Inkonsistenz auf die Definition des Produktes von Feld-

retisch gefunden: J. C. W $\mathrm{ARD}$, Phys. Rev. 78, 182 [1950]. Die erste allgemeine Untersuchung von Bedingungen für Matrixelemente, die aus dem Erhaltungssatz (28) folgen, stammt von G. Källen, Helv. Phys. Acta 26, 755 [1953]. 
operatoren am selben Ort geschoben werden kann. Man hätte dann im Sinn des am Ende von Abschn. I formulierten Konsistenzproblems die Rechnung solange mit einer durch Einbeziehung von $f$ veränderten Lagrange-Funktion zu wiederholen, bis volle Konsistenz vorliegt. Die in diesem Abschnitt studierten Identitäten können also nicht nur Aufschluß über die Konsistenz von Näherungen, sondern auch über die Struktur der Theorie bei kleinen Abständen geben.

Aus der obigen Ableitung folgen die Beziehungen (32), (33), (34) nur dann, wenn der Grundzustand bei der Transformation (30) invariant bleibt. Für Theorien mit entartetem Vakuum ist dies nicht mehr der Fall. Formal hätte man in $\delta V$ noch die Variation einzuschließen, die durch die Transformation des Vakuumzustandes bedingt ist. Dies führt jedoch da$z u$, da $\delta V$ identisch Null wird und gibt daher keine einschränkende Relation für $V$. Dies bedeutet jedoch nicht notwendig, daß bei entartetem Vakuum keine $\mathrm{W}_{\text {ARD-Identitäten gelten. }}$

\section{Massenoperator, Bethe-Salpeter-Gleichung}

Wir versuchen, für $G^{-1}$ einen expliziten Ausdruck zu erhalten, indem wir (19) in der Form

$$
\left(D+\varrho_{0} q+M\right)_{k l} G_{l m}=E_{k m}
$$

schreiben. Wenn (19) gilt, ist natürlich

$$
E_{k m}=\varrho_{0} \delta_{k m} \text {. }
$$

Der so eingeführte Massenoperator kann dann leicht durch $G$ und eine der Vierpunkfunktionen ausgedrückt werden. Es gilt z. B.

$$
\begin{aligned}
M_{k l} & =i V_{k r, n s}\left(2 G_{s r} \delta_{n l}-L_{n s, l r}\right) \\
& =i V_{k r, n s}\left(G_{s r} \delta_{n l}-G_{n m} \Gamma_{m s, l r}\right) .
\end{aligned}
$$

Wir fassen nun $M$ als Funktional von $G$ (und $q$ ) auf. Aus (2l) sehen wir, daß $M$ nur über $G$ von $q$ abhängt. Dies ist auch verständlich: $M$ hängt von den höheren Greesschen Funktionen ab, die über das ganze System wieder von $G$ abhängen. Es ist daher

$$
\frac{\delta M_{k l}}{\delta q_{p q}}=i K_{k s, l r} \frac{\delta G_{r s}}{\delta q_{p q}}\left(40 \text { a) mit } i K_{k s, l r}=\frac{\delta M_{k l}}{\delta G_{r s}} .\right.
$$

Durch Differentiation von (37) nach $q$ kann man daher eine Gleichung für eine Vierpunktfunktion erhalten ${ }^{10}$
$E_{h l} F_{l p, m q}=-\varrho_{0} G_{h q} G_{p m}-i G_{h k} K_{k j, l i} G_{l m} F_{i p, j q}$.

In einer kanonisch quantisierten Theorie ist $E$ der Einheitsoperator, und man erhält die bekannte Form der Gleichung. Wenn es Bindungszustände gibt, so $\mathrm{da} ß$ die Bilinearentwicklung (16) gilt, dann erfüllt jeder Term der Bilinearreihe (jede „Wellenfunktion“) die homogene Gleichung

$E_{i h} \varphi_{h j}(p, k)=-G_{i k}(p+k / 2)$
$\quad \cdot \int \frac{\mathrm{d}^{4} q}{(2 \pi)^{4}} K_{k s, l r}(p, q \mid k) G_{l j}(p-k / 2) \varphi_{r s}(q, k)$.

Definiert man eine homogene Boson-Fermion-Vertexfunktion durch

$$
\begin{aligned}
& \varphi_{i j}(p, k)=-G_{i k}(p+k / 2) \gamma_{k l}^{\mathrm{H}}(p+k / 2, p-k / 2) \\
& \cdot G_{l j}(p-k / 2), \quad(\varphi)=\text { ఫृ }
\end{aligned}
$$

so erfüllt auch diese die homogene Bethe-SalpeterGleichung. Die Emission des gebundenen Teilchens wird dann durch die Lösung der zugehörigen inhomogenen Gleichung (inhomogene Vertexfunktion) beschrieben, die an der Stelle $k^{2}=M_{n}^{2}$ einen Pol hat. Die Kopplungskonstante ist das Residuum dieses Poles, also im wesentlichen der Wert des Vertex auf der Massenschale $(\gamma \cdot p)=m, k^{2}=M_{n}{ }^{2}$.

Gl. (42) kann für die anderen Vierpunktfunktionen umgeschrieben werden. Für die Korrelationsfunktion ist der inhomogene Term komplizierter. Hingegen ist die Gleichung für $T$ besonders einfach

$E_{i j} T_{j k, l m}=K_{i k, l m}-i K_{i r, l s} G_{s p} G_{q r} T_{p k, q m}$.

Bei gegebenem $K$ ist dies, ebenso wie (41), eine lineare Integralgleichung für die Vierpunktfunktion. Kennt man die funktionale Abhängigkeit des Massenoperators $M$ von $G$, so kann $K$ durch Differentiation berechnet werden (dabei ist natürlich $G$ vor der Differentiation als willkürliche Funktion zu betrachten). Die tatsächliche Form dieser Abhängigkeit ist durch die Dynamik, d. h. den Wechselwirkungsausdruck bestimmt. Der Sinn der Einführung von $M[G]$ und der dadurch virtuell durchgeführten Auflösung des unendlichen Gleichungssystems der Greenschen Funktionen nach $G$ besteht darin, daß eine der Struktur von $V_{i k, l m}$ entsprechende Abhängigkeit wenigstens näherungsweise angegeben werden kann. Dies wird später gezeigt werden.

10 J. Schwinger, Proc. Nat. Acad. Sci. US 37, 452 [1951]. E. E. Salpeter u. H. A. Bethe, Phys. Rev. 84, 1232 [1951]. 
Das hier skizzierte Verfahren der Elimination der Funktionalableitung durch Auflösung kann noch weiter fortgesetzt werden: es ist möglich, auch noch $K$ gänzlich zu eliminieren, so daß man eine einzige, sehr komplizierte Gleichung übrig behält, die nur $G, T$ und seine Ableitungen nach $G$ enthält und nichtlinear ist. Die Struktur von Näherungsverfahren erscheint mit Hilfe dieser Gleichung durchsich- tiger ( siehe $^{2}$ ). Wir ziehen es vor, diese Prozedur an $L$ statt $T$ durchzuführen. Setzen wir in (21)

$$
\begin{aligned}
\frac{\delta L_{l s, n r}}{\delta q_{a b}} & =\frac{\delta L_{l s, n r}}{\delta G_{p q}} \frac{\delta G_{p q}}{\delta q_{a b}} \\
& =-\frac{\delta L_{l s, n r}}{\delta G_{p q}}\left(G_{p a} \delta_{j b}-L_{p b, j a}\right) G_{j q},
\end{aligned}
$$

so ist $\delta / \delta q$ ohne Einführung von $K$ eliminiert: die entstehende Gleichung für $L$ ist

$$
\begin{aligned}
& \left(D+\varrho_{0} q\right)_{k l} L_{l b, n a}+2 i V_{k r, l s}\left(G_{s r} L_{l b, n a}+\delta_{l n} G_{i r} L_{s b, i a}\right) \\
& \quad+i V_{k r, l s} L_{l s, i r} L_{i b, n a}-i V_{k r, l s}\left(G_{p a} \delta_{j b}+L_{p b, j a}\right) G_{j q} \frac{\delta L_{l s, n r}}{\delta G_{p q}}=2 i V_{k r, l n} G_{l a} G_{b r} .
\end{aligned}
$$

Diese Gleichung ist im Prinzip zusammen mit (20) zu lösen. Das System ist wesentlich einfacher als das aus (37) und der entsprechenden Gleichung für $T$ bestehende. Ebenso wie dieses letztere oder das System (37), (41) mit (10) und (40) kann auch das Gleichungspaar (20), (46) als Basis eines iterativen Näherungsverfahrens dienen.

Bei kritischer Betrachtung von (37) - (44) ist ersichtlich, daß diese Gleichungen an mehreren Stellen den Faktor $\varrho_{0}$ enthalten. Wenn $\varrho_{0}$ endlich ist, ist dies bloß ein Schönheitsfehler. Durch die Substitution

$$
\psi=\sqrt{\varrho} \psi^{\prime}, \quad l^{2}=\frac{1}{\varrho_{0}} l^{\prime 2}, \quad q=\frac{1}{\varrho_{0}} q^{\prime}
$$

kann die Theorie in eine kanonisch quantisierte verwandelt werden, denn die gestrichenen Größen erfüllen dieselben Beziehungen wie die ungestrichenen mit $\varrho_{0}=1$. Dieses Verfahren ist jedoch für $\varrho_{0}=0$ oder $\infty$ problematisch. Wir ziehen es deshalb vor, in den weiteren Abschnitten $\varrho_{0}$ (bzw. E) mitzunehmen.

Bei nichtkanonischer Quantisierung $\left(\varrho_{0}=0\right)$ ist $\left(D+\varrho_{0} q+M\right)$ nicht mehr das Inverse zu $G$. Durch Anwendung dieses Operators auf (41) oder (42) kann man zwar auch in diesem Fall eine (differentielle) Bethe-Salpeter-Gleichung gewinnen. Diese hat jedoch nicht nur eine unhandliche, weil unsymmetrische Form: infolge des Fehlens eines inhomogenen Terms sowohl in ihr als auch in (37) sind die üblichen Näherungsverfahren zur konsistenten Lösung des Systems nicht anwendbar. Die Einführung des Massenoperators erscheint daher nicht sinnvoll. Ein anderer Ausweg wäre der, daß man auf (38) verzichtet: da man $M$ jedenfalls nur näherungsweise angeben kann, wäre es möglich, daß (38) zwar exakt, nicht aber näherungsweise erfüllt ist. Man hätte dann Näherungen für $M$ und $E$ so anzugeben, daß alle betrachteten Gleichungen mit möglichst kleinem Fehler erfüllt sind, wobei geeignete Kriterien für die Kleinheit des Fehlers definiert werden müssen. Ein solches Verfahren wurde von Heisenberg ${ }^{1}$ vorgeschlagen und ist zur Berechnung von Masseneigenwerten geeignet. Für die hier betrachteten Konsistenzfragen kommt es jedoch weniger darauf an, einen analytischen Ausdruck für $M$ anzugeben, der in der Umgebung spezieller Werte von $p^{2}$ (z. B. in der Nähe von Masseneigenwerten) zu einer guten Näherung für $S^{-1}$ führt, sondern wir sind an der funktionalen Abhängigkeit $M[G]$ interessiert (und zwar sogar für beliebige $G$, da nach $G$ funktional differenziert werden muß). Wenn das Problem die Frage ist, ob $\varrho_{0}=0$ konsistent ist, darf aber diese Bedingung nicht vom gewählten Näherungsverfahren abhängig gemacht werden. Man könnte in der Tatsache, daß bei bisher vorgeschlagenen Realisierungen nichtkanonischer Zweipunktfunktionen ${ }^{11}$ der inverse Propagator nicht durch einen Differentialausdruck erster Ordnung gegeben ist, einen Hinweis darauf erblicken, daß für diesen Fall (37) bzw. (41) kein günstiger Ausgangspunkt ist. Möglicherweise ist daher für nichtkanonische Quantisierung das von (20), (46) ausgehende Verfahren vorzuziehen, das eine Alternative zu den oben erwähnten Näherungen für $M$ darstellt. Im Gegensatz zur Bethe-SAlpeterGleichung enthält die Gleichung für $L$ auch für $\varrho_{0} \rightarrow 0$ einen inhomogenen Term [die rechte Seite von (46) ], so daß iteriert werden kann. Der höhere Komplikationsgrad muß allerdings in Kauf genommen werden.

11 Dies sind die Dipolgeist-Regularisierung, die insbesondere in l. c. ${ }^{1}$ ausführlich diskutiert wird, und die Regularisierung durch asymptotische Skaleninvarianz: H. Mitter, Nuovo Cim. 32, 1789 [1964]. 


\section{Symmetrieverletzung und Goldstone- Teilchen}

Bei vielen quantenmechanischen Problemen ist der Grundzustand weniger symmetrisch als die Bewegungsgleichung. Für die Feldtheorie würde dies bedeuten, daß der Vakuumzustand nicht bei allen Transformationen, die (1) invariant lassen, ungeändert bleibt. Diese Symmetrieverletzung hat in der Elementarteilchentheorie Bedeutung erlangt, da sie es ermöglicht, einen Teil der in der Natur beobachteten exakten oder approximativen Symmetrien auf Eigenschaften des Vakuums abzuwälzen und dadurch mit einfacheren Feldgleichungen auszukommen. Im allgemeinen resultiert als Konsequenz das Auftreten von Polen bei der Ruhemasse Null ${ }^{12}$. Die Frage, ob diese Pole als physikalisch brauchbare $\mathrm{Zu}$ stände (Teilchen) interpretierbar sind und welche Eigenschaften sie haben, ist für die Theorie von Wichtigkeit. Im Anschluß an die im vorigen $\mathrm{Ab}$ schnitt verwendeten Methoden kann eine Formulierung dieses Problemkreises angegeben werden, die für die Klärung dieser Frage, auch im Rahmen von Näherungen, besonders geeignet erscheint ${ }^{13}$.

Wir nehmen an, daß die zur verletzten Symmetrie gehörige Gruppe eine Eichgruppe erster Art ist und studieren daher eine Transformation (30) mit konstanter Phase

$$
\psi \rightarrow e^{i \lambda B} \psi .
$$

Außerdem nehmen wir an, daß die im Massenoperator vorliegende Abhängigkeit von $G$ die Symmetrie erhält. Dieser Sachverhalt wird immer vorliegen, wenn diese Abhängigkeit in systematischer Weise gewonnen wird, denn sie entspricht dann im wesentlichen der Struktur des Wechselwirkungsausdruckes $V_{i k, l m}$.

Wir berechnen die durch (48) bewirkte Änderung von $G$ und variieren (37) :

$$
\delta M_{k l} G_{l m}+\left(D+\varrho_{0} q+M\right)_{k l} \delta G_{l m}=0 .
$$

Infolge der Symmetrie von $M[G]$ erfolgt die Änderung von $M$ nur durch $G$

$$
\delta M_{k l}=\frac{\delta M_{k l}}{\delta G_{i j}} \delta G_{i j}=i K_{k j, l i} \delta G_{i j} .
$$

12 J. Goldstone, Nuovo Cim. 19, 154 [1960]. - J. Goldstone, A. Salam u. S. W Winberg, Phys. Rev. 127, 965 [1962]. S. Bludman u. A. Klein, Phys. Rev. 131, 2364 [1963].

13 Diese Betrachtungsweise wurde von Johnson im Zusammenhang mit elektrodynamischen Problemen verwendet (mündl. Mitteilung, siehe auch M. BAKER et al., l. c. ${ }^{4}$ ).

14 Zur Normierung von Lösungen der Bethe-SALPETER-Gleichung mit Hilfe eines Erhaltungssatzes für den Strom $\delta G$ erfüllt daher die Gleichung

$$
\left(D+\varrho_{0} q+M\right)_{k l} \delta G_{l m}+i K_{k j, l i} G_{l m} \delta G_{i j}=0
$$

oder anders geschrieben

$$
E_{h l} \delta G_{l m}=-i G_{h k} K_{k j, l i} G_{l m} \delta G_{i j} .
$$

Durch Transformation in den Impulsraum sieht man, $\mathrm{da} ß$

$$
\frac{1}{\lambda} \delta G_{i j}=i(B G-G B)_{i j}
$$

eine spezielle Lösung $\varphi_{i j}^{(0)}(p, 0)$ der homogenen Bethe-Salpeter-Gleichung (42) zur Energie Null ist

$$
\begin{aligned}
E_{h i} \delta S(p)_{h l} & =-i S_{h k}(p) \\
& \cdot \int \frac{\mathrm{d}^{4} q}{(2 \pi)^{4}} K_{k j, l i}(p, q \mid 0) S_{l m}(p) \delta S_{i j}(q)
\end{aligned}
$$

Wenn das Vakuum bei der Transformation (48) invariant ist, wird diese Gleichung trivial erfüllt, denn dann verschwindet $\delta S$. Das macht man sich am besten an einem Beispiel klar: für gebrochene $\gamma_{5}$-Invarianz ist z. B. $\delta S$ im wesentlichen der Antikommutator von $S$ mit $\gamma_{5}$, der bei invariantem Vakuum verschwindet.

Wenn die Symmetrie durch den Grundzustand verletzt wird, ist $\varphi^{(0)}=(1 / \lambda) \delta S$ eine nichttriviale Lösung. Es ist dann zu untersuchen, ob diese Lösung als Wellenfunktion eines Teilchens interpretiert werden kann. Zunächst muß dazu die Frage beantwortet werden, ob $\varphi^{(0)}$ wirklich zur Masse Null $\left(k^{2}=0\right)$ gehört. Wenn $\varphi^{(0)}$ die Gleichung nur für $k_{\mu}=0$, nicht aber für $k_{\mu} \neq 0, k^{2}=0$ löst, ist es als Wellenfunktion eines Spurions zu interpretieren, das keine LoRENTz-Eigenschaften hat (es transformiert sich bei Lonentz-Transformationen wie das Vakuum). Wenn es erlaubt ist, die Integralgleichung (52) durch Rotation der $q_{0}$-Achse in eine Gleichung im euklidischen Raum zu transformieren, folgt aus der Lösbarkeit für $k_{\mu}=0$ diejenige für $k^{2}=0$ und es liegt kein Spurion vor. Als nächstes ist dann zu entscheiden, ob die mit der zu (42) gehörigen Vorschrift gebildete Norm ${ }^{14}$ von $\varphi^{(0)}$ (es handelt sich im wesentlichen um das Quadratintegral von $S \cdot \varphi^{(0)}$ ) endlich ist. Wenn dies nicht der Fall ist, hat man es mit

siehe K. Nishijima, Progr. Theor. Phys. 12, 279 [1954] u. ibid. 13, 305 [1955], S. Mandelstam, l. c. ${ }^{3}$, A. Klein u. C. Zемach, Phys. Rev. 108, 126 [1957]. - Eine andere Methode, die ohne Erhaltungssatz auskommt, stammt von R. E. Cutrosky u. M. Leon, Phys. Rev. 135, B 1445 [1964].

- Für eine ausführliche Diskussion des Falles $k^{2}=0$ siehe N. Nakanishi, Phys. Rev. 138 B, 1182 [1965]. 
einer anomalen Lösung zu tun (vgl. Abschnitt II, insbes. ${ }^{4}$ ). Mitunter ist in diesem Fall die zugehörige inhomogene Vertexfunktion nicht nur für $k^{2}=0$, sondern auch für endliche $k^{2}$ singulär (siehe BAKER et al., l. c. $\left.{ }^{4}\right)$. Es erhebt sich die Frage, ob die Existenz anomaler Lösungen eine Eigenschaft der Näherung oder davon unabhängig ist. Neue Untersuchungen ${ }^{14}$ machen letzteres wahrscheinlicher. Ist die Norm jedoch endlich, so handelt es sich um ein echtes Teilchen. Seine Kopplungskonstante kann, wie im vorigen Abschnitt angedeutet wurde, aus der entsprechenden Vertexfunktion berechnet werden. Man sieht, daß die hier angedeuteten Überlegungen für einen (z. B. näherungsweise) gegebenen Kern $K$ wirklich durchführbar sind.

\section{Exakte formale Lösung}

Mit Hilfe der hier verwendeten Funktionalmethoden läßt sich sogar ein geschlossener Ausdruck für die Transformationsfunktion $V$ [siehe (6) ] angeben, der de facto die formale Summierung der Störungsreihe darstellt. Man gewinnt ihn durch Vergleich des Ausdruckes für $V$ in den Wechselwirkungsbildern bezüglich der Nichtlinearität und bezüglich der Kopplung an die Quelle. Seien die entsprechenden LAGRANGE-Funktionen

$$
L_{Q}=q_{k l} \psi_{k l} \bar{\psi}_{k}, \quad L_{\mathrm{W}}=\frac{1}{2} V_{k l, r s} \bar{\psi}_{k} \bar{\psi}_{l} \psi_{s} \psi_{r},
$$

so ist, wenn wir die Anwesenheit der Quelle durch Q, die der Nichtlinearität durch $\mathrm{W}$ andeuten,

$$
\begin{aligned}
V \equiv\langle\text { out }| \text { in }\rangle_{\mathrm{Q}, \mathrm{W}} & =\frac{1}{N}\left\langle\text { out }\left|T e^{i L_{\mathrm{Q}}}\right| \text { in }\right\rangle_{\mathrm{W}} \\
& =\frac{1}{N^{\prime}}\left\langle\text { out }\left|T e^{i L_{\mathrm{W}}}\right| \text { in }\right\rangle_{\mathrm{Q}},
\end{aligned}
$$

wobei die Normierungsfaktoren die Transformationsfunktionen ohne Quelle bzw. ohne Nichtlinearität sind

$$
\left.N=\langle\text { out }| \text { in }\rangle_{\mathrm{W}}, \quad N^{\prime}=\langle\text { out }| \text { in }\right\rangle_{\mathrm{Q}} .
$$

Entwickeln wir nun $V$ in der ersteren Darstellung nach Potenzen von $l^{2}$ und vergleichen mit der zweiten Form, so sehen wir

15 Eine explizite Berechnung von $N^{\prime}$ für eine lokale Quelle, die auf den hier vorliegenden Fall sofort übertragbar ist, findet man bei J. Schwinger, The Theory of Coupled Fields, Harvard Lecture 1954. (unveröffentlicht), dem wir auch in den übrigen Ableitungen dieses Abschnittes folgen. Siehe auch Y. Katayama, Progr. Theor. Phys. 7, 205 [1952];

$$
\begin{aligned}
V & =(V)_{l^{2}=0}+l^{2}\left(\frac{\partial V}{\partial l^{2}}\right)_{l^{2}=0}+\ldots \\
& =\frac{1}{N^{\prime}}\left(N^{\prime}+\frac{i}{2} V_{k l, r s}\left(\frac{\delta}{\delta q_{k s}} \frac{\delta}{\delta q_{l r}} V\right)_{l^{2}=0}+\ldots\right. \\
& =\frac{1}{N^{\prime}}\left(N^{\prime}+\frac{i}{2} V_{k l, r s} \frac{\delta}{\delta q_{k s}} \frac{\delta}{\delta q_{l r}} N^{\prime}+\ldots\right) .
\end{aligned}
$$

Durch Summation erhält man

$$
V=\frac{1}{N^{\prime}} \exp \left\{\frac{i}{2} V_{k l, r s} \frac{\delta}{\delta q_{k s}} \frac{\delta}{\delta q_{l r}}\right\} N^{\prime} .
$$

Damit ist $V$ auf die Transformationsfunktion $N^{\prime}$ einer Theorie ohne Wechselwirkung, aber mit Quellen zurückgeführt. Da die Greesschen Funktionen durch funktionale Differentiation aus $V$ gewonnen werden können, sind sie durch die entsprechenden der freien Theorie (mit Quellen) ausdrückbar. Die letzteren sind aber mit Hilfe der Zweipunktfunktion

$$
G_{k e}^{(0, q)}=\frac{\delta \ln N^{\prime}}{\delta q_{l k}}
$$

als Slater-Determinanten darzustellen, z. B.

$$
G_{(0, q)}^{k l, m n}=\left|\begin{array}{ll}
G_{k m}^{(0, q)} & G_{k n}^{(0, q)} \\
G_{l m}^{(0, q)} & G_{l n}^{(0, q)}
\end{array}\right| .
$$

Alle Greenschen Funktionen der wechselwirkenden Theorie können also im Prinzip durch die Zweipunktfunktion (56) ausgedrückt werden. Für $V$ lautet dieser Sachverhalt ${ }^{15}$

$$
\begin{aligned}
V= & \frac{1}{\operatorname{det}\left(1+\varrho_{0}^{\prime} q G^{(0)}\right)} \\
& \cdot \exp \left\{\frac{i}{2} V_{k l, r s} \frac{\delta}{\delta q_{k i}} \frac{\delta}{\delta q_{l r}}\right\} \operatorname{det}\left(1+\varrho_{0}^{\prime} q G^{(0)}\right) .
\end{aligned}
$$

Dabei ist $G^{(0)}$ der Propagator eines freien Teilchens (ohne Quelle)

$$
G_{k l}^{(0)}=\left(D^{-1}\right)_{k l} .
$$

$Q_{0}^{\prime}$ ist das erste Spektralmoment von (56) und die Multiplikation bei der Determinantenbildung schließt (im Sinne des in I eingeführten Matrizenkalküls) Raumintegration und Spin- bzw. Isospinsummation ein.

Die exakte Lösung (58) ist im allgemeinen für praktische Rechnungen zu kompliziert. Sie kann jedoch zum Nachweis von allgemeinen Eigenschaften der Greenschen Funktionen dienen. Die Störungs-

K. Yамаzaki, ibid. 7, 449 [1952] ; S. Hori, ibid. 7, 578 [1952] ; S. F. Edwards u. R. E. Peierls, Proc. Roy. Soc. London A 224, 24 [1954] ; K. SyмanziK, Z. Naturforschg. 9 a, 809 [1954] ; I. M. Gelfand u. R. A. Minlos, Dokl. Nauk USSR 97, 209 [1954] ; E. S. FradKIN, ibid. 98, 47 [1954]. 
reihe erhält man durch Entwicklung der Exponentialfunktion. Wenn man als Modell eine Theorie im zweidimensionalen Raum betrachtet, kann (55) sogar explizit ausgewertet werden ${ }^{16}$, da der Propagator (56) dann eine einfache Form hat.

Unklar ist an der obigen Formulierung noch, welche Quantisierung $\varrho_{0}^{\prime}$ man in $N^{\prime}$ bei nichtkanonischem $\varrho_{0}$ zu verwenden hat. Da es sich um eine freie Theorie handelt, wäre a priori kein Grund dafür vorhanden, die kanonische Quantisierung abzulehnen. Die Entwicklung des Exponentials in (55) bzw. (58) führt dann Term für Term auf eine kanonische, aber divergente Theorie. Ob die Summierung beide Tatsachen ändert und den radikalen Unterschied zwischen $\varrho_{0}$ und $\varrho_{0}^{\prime}$ herbeiführt, ist nicht sicher. Es wäre auch denkbar, daß man nur zu einer widerspruchsfreien Formulierung einer nichtkanonisch quantisierten Theorie gelangt, wenn man von einer klassischen Lösung $\left(\varrho_{0}^{\prime}=0\right)$ ausgeht [die formale Schwierigkeit, daß dann das Argument der Determinante in (58) trivial wird, kann durch Betrachtung der Greenschen Funktionen vermieden werden]. Diese Zusammenhänge könnten an zweidimensionalen Modellen studiert werden.

\section{Konsistente Näherungsverfahren}

Ein mit der Existenz lokaler Ströme verträgliches Näherungsverfahren ist in der Theorie des Mehrkörperproblems ${ }^{17}$ und in der Quantenelektrodynamik ${ }^{18}$ verwendet worden und kann auf den hier vorliegenden Fall übertragen werden, weist aber bei nichtkanonischer Quantisierung die am Ende von VI erwähnte Schwierigkeit auf. Das Verfahren besteht im wesentlichen in einer Entwicklung von $M$ nach Potenzen der Wechselwirkung $V_{i k, l m}$, wobei aber $M$ stets als Funktional der exakten Zweipunktfunktion aufgefaßt wird. Aus der Störungsreihe für $M$ kann diese Entwicklung gewonnen werden, indem alle Selbstenergieteile summiert und durch die exakte Zweipunktfunktion ersetzt werden, so daß $M$ die Summe über alle Skelettgraphen ist. Jedes Glied dieser Summe entsteht also durch Summation von unendlich vielen Termen der Störungsreihe. Ohne Bezugnahme auf die Störungstheorie kann diese Entwicklung von $M$ in folgender Weise hergeleitet wer-

16 Ch. M. Sommerfield, Ann. Phys. N. Y. 26, 1 [1963].

17 Siehe z. B. L. P. Kadanoff u. G. Baym, Quantum Statistical Mechanics, Benjamin Inc., New York 1962, Kap. 5. den: Man berechnet durch Differtiation der DysonGleichung (37) $\delta G / \delta q$ und setzt dies in die durch Vergleich von (19) und (37) entstehende Beziehung zwischen $M$ und $\delta G / \delta q$ ein. So erhält man eine Funktionaldifferentialgleichung für $M$

$$
M_{k l}=2 i V_{k r, l s} G_{s r}-\frac{1}{\varrho_{0}} V_{k r, n s} G_{n m} \frac{\delta M_{m l}}{\delta q_{r s}} .
$$

Diese Gleichung wird durch eine konsequente Reihenentwicklung nach Potenzen von $V_{k l, m n}$ iterativ gelöst. Die beiden ersten Terme sind

$M_{k l}=2 i V_{k r, l s} G_{s r}-\frac{1}{\varrho_{0}} V_{k r, l s} G_{l i} V_{i q, j p} G_{p r} G_{s q}+\ldots$

In graphischer Schreibweise ist die Struktur klarer. Für die Wechselwirkung $(4 \mathrm{~b})$ erhalten wir

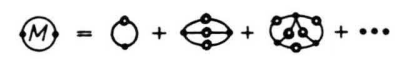

Differenziert man die mit diesem Massenoperator gewonnene Funktion $G^{-1}$ nach $q$, so erhält man den zugehörigen Vertex $T$. Dieser erfüllt die BEтHESalpeter-Gleichung [vgl. (41)]

$$
\Gamma_{k m, l q}=\delta_{k q} \delta_{p l}-\frac{i}{\varrho_{0}} K_{k j, l i} G_{i r} \Gamma_{r p, s q} G_{s j},
$$

wobei $K$ eine Summe von Termen ist, die aus den einzelnen Summanden von $M$ gemäß $(40 \mathrm{~b})$ entstehen

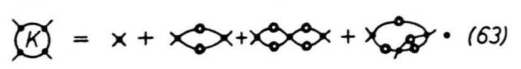

Bricht man sowohl die Entwicklung für $M$ als auch die für $K$ an der gleichen Stelle ab, so erhält man stets ein System von Gln. (37), (62) für $G$ und $\Gamma$, das die WARD-Identität (36) erfüllt, wobei aber

$$
f(z)=\frac{1}{\varrho_{0}} \delta(z)
$$

ist. Um die Theorie konsistent zu machen, hat man daher (vgl. S. 1511) die ganze Untersuchung mit einem abgeänderten Wechselwirkungsausdruck noch einmal durchzuführen, der diesem $f$ entspricht. Das bedeutet praktisch, daß man die "Renormierung“ (47) durchführt und de facto $\varrho_{0}=1$ setzt. Die Konsistenzforderung verbietet also bei diesem Näherungsverfahren nicht nur zu starke Nichtlokalität [weniger radikale Formen wie (32) sind noch zu-

18 K. Johnson, M. Baker u. R. Willey, Phys. Rev. 136 B, 1111 [1964]. 
lässig], sie erzwingt außerdem die kanonische Quantisierung. Ohne die einzelnen Näherungen zu berechnen, kann man dies auch in folgender Weise einsehen: Die W ARD-Identität kann unter alleiniger Benützung von (12) und der Eichkovarianzeigenschaft

$$
G_{k l}^{-1} \rightarrow \exp \left\{-i\left(\lambda_{k}-\lambda_{l}\right)\right\} G_{k l}
$$

abgeleitet werden. Für den aus (37) mit (61) berechneten Ausdruck für $G^{-1}$ ist (12) erfüllt, wenn der Vertex in der entsprechenden Näherung aus (62) berechnet wird. Die Eichkovarianzeigenschaft für $G^{-1}$ gilt ebenfalls Term für Term, aber nur, wenn ent- weder $\varrho_{0}=1$ ist oder $M_{k l}$ einen Term $\left(\varrho_{0}-1\right) D_{k l}$ enthält. Da in der Entwicklung (61) ein solcher Term nicht auftritt, führt nur $\varrho_{0}=1$ zur Konsistenz.

Das eben beschriebene Verfahren kann aber noch weiter verbessert werden, ohne daß die Konsistenz verlorengeht. Wie aus (63) ersichtlich ist, treten in den höheren Näherungen zu $K$ Glieder auf, die Iterationen niedrigerer Terme sind $(\mathrm{z}$. B. ist der dritte Term die Iteration des zweiten). Auch diese Terme können auf verhältnismäßig durchsichtige Weise summiert werden. $\mathrm{Zu}$ diesem $\mathrm{Zweck}$ schreiben wir Gl. (20) als Beziehung zwischen $G$ und $T$

$$
\left(D_{k l}+\varrho_{0} q_{k l}+i V_{k r, j s}\left(G_{s r} \delta_{j l}-G_{j n} \Gamma_{n s, l r}\right)\right) G_{l m}=\varrho_{0} \delta_{k m}
$$

und definieren sukzessive Approximationen zu $G$ und $I$ durch folgende Prozedur:

$$
\left(D+\varrho_{0} q\right)_{k l}+i V_{k r, j s}\left(G_{s r}^{(n)} \delta_{j l}-G_{j n}^{(n)} \Gamma_{n s, l r}^{(n-1}\right)=E_{k h} G_{n l}^{(n)-1} .
$$

Bei gegebenem $\Gamma^{(n-1)}$ ist dies eine Gleichung für $G^{(n)}$. Nach Lösung dieser Gleichung berechnen wir die nächste Näherung zu $T$ mit Hilfe der durch Differentiation von (66) nach $q$ entstehenden Gleichung

$$
E_{k h} \Gamma_{n p, l q}^{(n)}=\varrho_{0} \delta_{k q} \delta_{l p}+i V_{k r, j s}\left(-\delta_{j l} G_{s t}^{(n)} \Gamma_{t p, v q}^{(n)} G_{v r}^{(n)}+G_{j n}^{(n)} \Gamma_{n p, m q}^{(n)} G_{m i}^{(n)} \Gamma_{i s, l r}^{(n-1)}-G_{i j}^{(n)} \frac{\delta \Gamma_{i s, l r}^{(n-1)}}{\delta q_{q p}}\right) .
$$

Als niedrigste Näherung ist

$$
I_{k p, l q}^{(0)}=\delta_{k q} \delta_{l p}
$$

zu nehmen. Man sieht, daß in dieser Weise in jeder Näherung eine Gleichung vom Bethe-SALPEter-Typ (62) für $T$ entsteht, deren Kern gemäß $(40 \mathrm{~b})$ gebildet ist. Ordnung für Ordnung ist auch

$$
\Gamma_{i p, j q}^{(n)}=\frac{\delta G_{i j}^{(n)-1}}{\delta q_{q p}}
$$

erfüllt, so daß auf die Gültigkeit von (36) mit (64) wie oben geschlossen werden kann. Graphische Analyse zeigt, daß die niedrigste Näherung mit der entsprechenden des vorherigen Verfahrens identisch ist, hingegen in der nächsten in $K$ bereits alle Kettenglieder („Z̈̈pfe“)

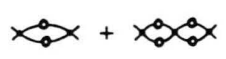

aufsummiert sind. Man erhält somit bei Einsetzen des so gefundenen $T$ in die Dyson-Gleichung (37), also bei Berechnung von $G^{(2)}$, bereits die von ,gebundenen Paaren" herrührenden Korrekturen zu $G$ : wenn es solche Bindungszustände gibt, wird man erwarten, daß $\Gamma^{(1)}$ die entsprechenden Pole hat.

In niedrigster Näherung $(n=1)$ erhält man den freien Propagator, da der Hartree-Term aus Invarianzgründen verschwindet ${ }^{19}$. Die Gleichung für die Vertexfunktion ist eine Integralgleichung mit der symbolischen Lösung

$$
\Gamma^{(1)}=\frac{1}{1-V S^{(1)} S^{(1)} V} .
$$

Die Lösung kann sogar explizit angegeben werden, da die Gleichung für $F$ (im Impulsraum) wegen der Lokalität der Wechselwirkung eine algebraische Gleichung ist. Die Lösung enthält jedoch Divergenzen, denn die in ihr auftretende charakteristische Größe

$$
\begin{aligned}
& V S S V \sim H_{\mu \nu}(k) \\
& \sim \operatorname{Sp} \int Q^{\mu} S(p+k / 2) Q^{\nu} S(p-k / 2) \mathrm{d}^{4} p
\end{aligned}
$$

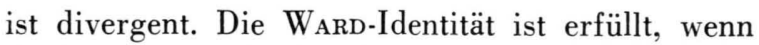
(64) gilt und außerdem $H_{\mu \nu}$ transversal ist

$$
H_{\mu \nu}(k)=\left(g_{\mu \nu}-\frac{k_{\mu} k_{v}}{k^{2}}\right) h\left(k^{2}\right) .
$$

Diese Bedingung ist wegen der starken Divergenz des Integrals nicht erfüllt. Wenn man daher ein gefährliches Verfügen über divergente Ausdrücke vermeiden will, muß man diese Inkonsistenz durch $\mathrm{Ab}$ änderung von $V_{i k, l m} \mathrm{zu}$ beheben suchen. Einen Hinweis darauf, wie dies geschehen könnte, gibt die ent-

19 Dies ist nicht mehr der Fall, wenn man die Lorentz-Invarianz des Vakuums aufgibt. Für diesen Fall siehe J. D. Bjorken u. G. S. Guralnik, l. c. ${ }^{6}$. 
sprechende Situation in der Quantenelektrodynamik. Untersucht man die nächste Näherung in (66) für den Propagator und interpretiert sie im Sinn von Abschnitt IV, so sieht man, daß $a_{k l}$ dem HartreeTerm entspricht und durch die niedrigste Störungsnäherung ersetzt ist. $D$ ist im wesentlichen durch $V \cdot F^{(1)}$ gegeben. Das Integral $H$ entspricht der „Photonselbstenergiekorrektor“ (mit der es auch analytisch weitgehend übereinstimmt), die dieselbe Schwierigkeit hinsichtlich der Transversalität aufweist (quadratisch divergente Photonmasse). Verwendet man jedoch in der Elektrodynamik an Stelle von (27) einen Ausdruck der Form (31), so wird diese Schwierigkeit vermieden: In der Gleichung für $D$ treten als Folge der Exponentialfunktion in (31) zusätzliche Terme auf, die für die Transversalität sorgen und außerdem den Divergenzgrad verringern ${ }^{20}$. Es ist daher zu erwarten, daß die Verwendung solcher Stromausdrücke hier ähnliche Effekte hat. Eine genaue Untersuchung dieses Sachverhaltes ist anscheinend noch nicht erfolgt.

\section{Näherungen für $L$}

Durch geeignete Approximation von $L$ und Einsetzen in die Dyson-Gleichung in der Form (20) lassen sich ebenfalls Näherungsmethoden gewinnen. Ein mit der $W_{\text {ARD-Identität konsistentes Verfahren }}$ könnte z. B. analog wie im vorigen Abschnitt gewonnen werden: man schreibt Gl. (20) als Beziehung zwischen $G^{(n)}$ und $L^{(n-1)}$ und ersetzt (21) durch jene Gleichung, die man durch Differentiation dieser Beziehung nach der Quelle erhält. In nullter Näherung erhält man dann wieder den freien Propagator und das Verfahren ist äquivalent $\mathrm{zu}$ dem im vorigen Abschnitt betrachteten.

Eine der Struktur nach völlig andere Näherung erhält man, wenn man in (21) oder (46) einzelne Terme wegläßt und die so gewonnene Gleichung zusammen mit (20) löst. Wie eine solche Prozedur zu einem systematischen Verfahren ausgebaut werden kann, ist ein kompliziertes Problem und soll deshalb hier nicht behandelt werden. Wir wollen jedoch kurz einige Eigenschaften der einfachsten möglichen Näherung diskutieren, soweit dies ohne allzu detaillierte Rechnung möglich ist.

20 Siehe z. B. K. Johnson, Quantum Electrodynamics, Brandeis Summer Institute lecture 1964, Prentice Hall, Inc. 1965.

21 Sie entsteht dort aus der niedrigsten nichttrivialen $\mathrm{T}_{\text {АMм- }}$ Dancoff-Näherung durch Vernachlässigung einiger Terme („verkürzte“ TAmm-Dancoff-Methode). Eine Verbesse-
Wir vernachlässigen in $(21)$ :

(a) den nichtlinearen Term,

(b) die Funktionalableitung von $L$,

(c) den linearen Term $V \cdot L$.

Dann kann $L$ sofort berechnet werden. Wir erhalten

$$
L_{k b, n a}^{(1)}=2 i G_{k j}^{(0, q)} V_{j r, l n} G_{l a}^{(1)} G_{b r}^{(1)}
$$

und damit für die unterste Näherung zu $M$

$M_{k l}^{(1)}=2 i V_{k r, l s} G_{s r}^{(1)}-2 V_{k r, n s} G_{n i}^{(0, q)} V_{i p, l q} G_{q s}^{(1)} G_{s p}^{(1)}$.

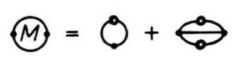

Durch Einsetzen in (20) entsteht eine nichtlineare Integralgleichung für $G^{(1)}$

$$
\begin{aligned}
& \left(\left(D+\varrho_{0} q\right)_{k l}+2 i V_{k r, l s} G_{s r}^{(1)}\right. \\
& \left.\quad-2 V_{k r, n s} G_{n i}^{(2, q)} V_{i p, l q} G_{q r}^{(1)} G_{s p}^{(1)}\right) G_{l m}^{(1)}=\varrho_{0} \delta_{k m} .
\end{aligned}
$$

Diese Näherung ist in der von Heisenberg und Mitarbeitern studierten Theorie ${ }^{1}$ in einem ähnlichen $\mathrm{Zu}$ sammenhang verwendet worden ${ }^{21}$. Dabei handelte es sich um eine schwächere Form eines Konsistenzproblemes: Führt man nämlich in (20) Zwischenzustände ein und verwendet die Translationsinvarianz, so erhält man die Gleichung für jede FourierKomponente von $G_{l m}$. Für jeden Fermionenzustand muß $G$ einen Pol haben und daher $(D+M)$ verschwinden. In den erwähnten Arbeiten war dieser Sachverhalt für eine plausibel angenommene Zweipunktfunktion überprüft worden. Stellt man die Forderung nach Konsistenz in schärferer Weise, so wird man (71) als Bestimmungsgleichung für $G^{(\mathrm{t})}$ auffassen. Sofern die Gleichung nichttriviale Lösungen hat, muß es sich um regularisierte Lösungen handeln, d. h. die ersten beiden Momente von $G$ müssen verschwinden

$$
\int \varrho\left(x^{2}\right) \mathrm{d} x^{2}=\int x^{2} \varrho\left(x^{2}\right) \mathrm{d} x^{2}=0,
$$

da andernfalls die im nichtlinearen Glied auftretenden Produkte nicht existieren. Da die Gleichung formal skaleninvariant ist, könnte man einen Ansatz mit dem in l. c. ${ }^{11}$ untersuchten asymptotischen Verhalten für kleine Abstände versuchen. Praktisch wird die Frage, ob vernünftige Lösungen existieren, wahrscheinlich nur durch numerische Analyse geklärt werden können. Wenn dies der Fall ist, bleibt immer

rung, die einen Teil dieser Terme berücksichtigt und der Mitnahme eines Teiles der linearen Terme (c) entspricht, wurde ebenfalls verwendet: H. P. DürR u. W. Heisenberg, Nuovo Cim. 37, 1446 [1965]. 
noch die Frage nach der Konsistenz mit der $\mathrm{W}_{\text {ARD }}$ Identität offen. Es ist klar, daß (35) nicht mit der aus (69) mit Hilfe der allgemeinen Beziehung [vgl. (13) und (14) ]

$$
F_{i k, l m}=-G_{i m} G_{k l}-L_{i k, j m} G_{j l}
$$

berechneten Vierpunktfunktion erfüllt sein kann, denn die so gefundene Funktion $F^{(1)}$ wäre nicht die Funktionalableitung von $G^{(1)}$ nach der Quelle. Eine Chance für Konsistenz besteht hingegen, wenn man als zugehöriges $F$ die Lösung der aus (71) durch Differentiation entstehenden Bethe-Salpeter-Gleichung

$$
\begin{aligned}
& \left(D+\varrho_{0} q+M^{(1)}\right)_{k l} F_{l s, m r}^{(2)}+\frac{\delta M_{k l}^{(1)}}{\delta G_{i j}^{(1)}} F_{i s, j r}^{(2)} G_{l m}^{(1)} \\
& +\varrho_{0}\left(\delta_{k r} \delta_{l s}-\frac{\delta M_{k l}^{(1)}}{\delta G_{i j}^{(0)}} G_{i r}^{(0, q)} G_{s j}^{(0, q)}\right) G_{l m}^{(1)}=0
\end{aligned}
$$

nimmt (der letzte Term tritt auf, weil $M^{(1)}$ in dieser Näherung auch über $G^{(0, q)}$ von der Quelle abhängt) . Dann ist die Differentialbeziehung (10) zwischen $F$ und $G$ auch in dieser Näherung erfüllt und es bleibt lediglich die Frage zu klären, ob die mit dem Auftreten von $\varrho_{0}$ zusammenhängenden Probleme (vgl. IX) der Konsistenz hier wirklich im Wege stehen. Dazu muß aber sowohl (70) als auch (74) wirklich gelöst werden.

Im Gegensatz zu der untersten Näherung des im vorigen Abschnitt behandelten Verfahrens ist (73) auch im Impulsraum eine echte Integralgleichung. Der Kern ist

$$
\begin{gathered}
K_{(1)}^{k j, l i}=2 V_{k j, l i}-2 V_{k j, n s} G_{s p}^{(1)} G_{n j}^{(0, q)} V_{r p, l i} \\
-2 V_{k s, n i} G_{p s}^{(1)} G_{n r}^{(0, q)} V_{r j, l p} . \\
\mathbb{K})=\times+\infty
\end{gathered}
$$

Eine ähnliche Integralgleichung erhält man ${ }^{22}$, wenn man das Bindungsproblem mit derselben ,verkürzten Tamm-Dancoff-Methode“ behandelt, die in der oben erwähnten schwächeren Fassung des Konsistenz-

$\cong 2$ H. Stumpf u. H. Yамамото, Z. Naturforschg. 20 a, 1 [1965]. problems für den Fermionzustand angewandt wurde. Sie unterscheidet sich strukturell von der zu (74) gehörigen homogenen BETHE-SALPETER-Gleichung für die aus $F^{(2)}$ nach (16) gebildeten Wellenfunktionen nur im Kontaktterm.

Eine Verfeinerung der hier betrachteten Näherung könnte erhalten werden, indem die nach (73) zu $F^{(2)}$ gehörige Funktion $L^{(2)}$ in $(20)$ verwendet wird. Man erhielte dann u. a. die Rückwirkung der Bindungszustände auf die Fermionen. Es ist jedoch zweifelhaft, ob es nicht günstiger wäre, zuerst in (21) bzw. (46) mehr Terme mitzunehmen. Die Konsistenzfrage läßt sich dann im Prinzip genau wie für die hier betrachtete unterste Näherung diskutieren.

\section{Schlußbemerkungen}

Mit Hilfe der hier untersuchten Methoden ist es gelungen, wesentliche Tatbestände der Feldtheorie, wie z. B. das Auftreten von Bindungszuständen und deren Ankopplung oder die Existenz von lokalen Erhaltungssätzen, auf analytische Eigenschaften bzw. Beziehungen zwischen wenigen Funktionen zurückzuführen, die ausnahmslos Vakuumerwartungswerte sind. Außerdem ermöglicht es der Funktionalkalkül, auch über kompliziertere Probleme wie die Struktur und Konsistenz von Näherungsverfahren Auskunft zu erhalten und den Überblick zu behalten. Als konkretere Resultate dürfen die in den Abschnitten IX und $\mathrm{X}$ formulierten Aussagen über die Konsistenz von verschiedenen in der Literatur verwendeten $\mathrm{Nä-}$ herungsverfahren gelten. Über den Nutzen der hier gemachten Vorschläge wird die detaillierte Rechnung in konkreten Fällen zu entscheiden haben, die durchführbar erscheint, aber noch aussteht.

Ich danke den Herren Prof. W. Heisenberg, Prof.

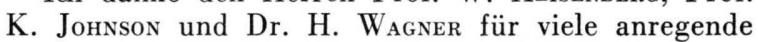
Diskussionen. 